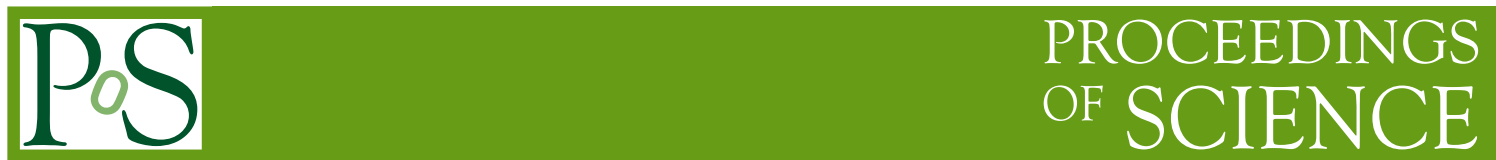

\title{
Eternally Inflating Multiverse and Many Worlds in Quantum Mechanics: Same Concept?
}

\author{
Yasunori Nomura* \\ Berkeley Center for Theoretical Physics, Department of Physics, University of California, \\ Berkeley, CA 94720, USA \\ Theoretical Physics Group, Lawrence Berkeley National Laboratory, CA 94720, USA \\ Kavli Institute for the Physics and Mathematics of the Universe (WPI), UTIAS, The University of \\ Tokyo, Kashiwa, Chiba 277-8583, Japan \\ E-mail: ynomura@berkeley.edu
}

\begin{abstract}
Recent observational and theoretical progress has led to the dramatic new view that our universe may be one of the many universes in which low energy physical laws take different forms: the multiverse. I describe impacts this view may have on our fundamental understanding of spacetime and gravity, especially the idea that the eternally inflating multiverse and quantum mechanical many worlds are the same thing. I will also mention possible future observations that may further probe the multiverse.
\end{abstract}

2nd World Summit: Exploring the Dark Side of the Universe 25-29 June, 2018 - EDSU2018

University of Antilles, Pointe-à-Pitre, Guadeloupe, France

\footnotetext{
*Speaker.
} 


\section{Do We Live in the "Multiverse"?}

It is possible that we live in the era of a major paradigm shift in cosmology. Specifically, we may be learning that what we thought of as the entire universe is only a tiny part of a much larger structure, called the "multiverse." In the multiverse, there are many universes in which what we once thought were fundamental laws of physics take different forms than those in our universe; for example, they may have different elementary particles with different properties and even different spacetime dimensions. This picture has been developed by many researchers over the past decades [1].

In view of the history, the idea that our universe is only a part of a much larger structure, by itself, is not particularly eccentric. A nontrivial thing is that our observational and theoretical understanding of nature seems advanced enough that we can now discuss this possibility scientifically. Observationally, we have learned more and more that we live in a highly special universe-it seems that the "physical laws" of our universe take such special forms that if they were changed slightly, there would be no interesting structure, let alone intelligent life [2]. Currently, the most significant example illustrating this comes from the value of the vacuum energy. In 1998, it was discovered that the vacuum energy density in our universe is nonzero and comparable to the energy density of the matter present today. This is very peculiar given that time dependence of the two densities are very different. It is hard to understand this fact without resorting to the cleverness of a creator, unless there are many universes with varying "physical laws," and we simply happen to emerge in a universe which allows for intelligent life to develop. Indeed, one can show that if vacuum energy were much larger than the observed value, then the universe would not contain any interesting structure [3]. In other words, if intelligent life develops and observes the universe, then it always finds that the vacuum energy density is not much different from the matter energy density.

Another striking fact is that our current understanding of fundamental physics seems to lead to the multiverse picture in a very natural way. Suppose at some point in the cosmic history, space was expanding exponentially fast. We knew, already in 80's, that such expansion-inflationis generically eternal. (This inflation is not the slow-roll inflation that has occurred within our universe.) During inflation, various non-inflating regions called bubble universes-of which our own universe could be one-may form, much like bubbles in boiling water. Since ambient space expands exponentially, however, these bubbles do not percolate; rather, the process of creating bubble universes lasts forever in an eternally inflating background. Now, recent progress in string theory suggests that physical laws describing the interiors of these bubble universes may differ bubble by bubble since the shape and size of extra compact dimensions-predicted by the theory and responsible for the form of the physical laws-may differ in each bubble [4]. This is exactly the multiverse setup needed to understand the "specialness" of our universe. It is intriguing that the properties of the fundamental theory that physicists once thought a nuisance-the eternal nature of inflation and the existence of extra dimensions in string theory-are exactly those needed to understand features of our universe as we observe it today.

\section{A Predictivity Crisis}

The version of the multiverse described above, called the eternally inflating multiverse, is 
very attractive. It is theoretically well motivated and has a potential to explain various features of our universe. The eternal nature of inflation, however, causes a serious problem with predictivity. Because the process of creating bubble universes occurs infinitely many times, "In an eternally inflating universe, anything that can happen will happen; in fact, it will happen an infinite number of times," as phrased by Alan Guth [5]. Suppose we want to calculate the relative probability for (any) events $A$ and $B$ to happen in the multiverse. Following the standard notion of probability, we might define it as the ratio of the numbers of times events $A$ and $B$ happen throughout the whole spacetime: $P=N_{A} / N_{B}$. In the eternally inflating multiverse, however, both $A$ and $B$ occur infinitely many times: $N_{A}, N_{B}=\infty$. This expression for the probability $P$, therefore, is ill-defined. One might think that this is merely a technical problem-perhaps we simply need to "regularize" to make both $N_{A, B}$ finite at a middle stage of the calculation to get a well-defined answer. This, however, is not the case. One finds that depending on the details of this regularization procedure, one can obtain any "prediction" one wants, and there is no a priori preferred way to proceed over others-predictivity of physical theory seems to be lost!

Over the past decades, some physicists and cosmologists have been thinking about many aspects of this so-called measure problem in eternal inflation. Many of these scientists, including myself, have the view that this problem offers a window into deep issues associated with spacetime and gravity. Indeed, we will see that a proper understanding of this issue seems to require a surprising, intrinsically quantum mechanical effect acting at large distances in a system with gravity. Before looking into this, however, we need to make a little detour to consider an apparently unrelated system: spacetime with a black hole.

\section{Quantum Mechanics in a System with Gravity}

Suppose we drop some book $A$ into a black hole and observe subsequent evolution of the system from a distance. The book will be absorbed into (the horizon of) the black hole, which will then eventually evaporate, leaving Hawking radiation. Now, let us consider another process of dropping a different book $B$, instead of $A$, and see what happens. The subsequent evolution in this case is similar to the case with $A$, and we will be left with Hawking radiation. However, this final-state Hawking radiation arising from $B$ is (believed by many to be) different from that arising from $A$ in its subtle quantum correlation structure, so that if we have perfect knowledge about the final-state radiation then we can reconstruct the original book. This property, unitarity, is considered to provide the correct picture for black hole dynamics. To recap, the information about the original book will not be lost—it will simply be distributed in final-state Hawking radiation in a highly scrambled form.

A puzzling thing occurs, however, if we observe the same phenomenon from the viewpoint of an observer who is falling into the black hole with a book. In this case, the Einstein's equivalence principle says that the book does not feel gravity (except for the tidal force which is tiny for a large black hole), so it simply passes through the black hole horizon without any disruption. This implies that all the information about the book-in fact, the book itself — will be inside the horizon at late times. On the other hand, we have just argued that from a distant observer's point of view, the information will be outside - first on the horizon and then in Hawking radiation. One might think that the information is simply duplicated: one copy inside and the other outside. This, however, 
cannot be the case. Quantum mechanics prohibits faithful copying of full quantum information, by the so-called no-cloning theorem. Therefore, it seems that the two pictures by the two observers cannot both be correct.

The proposed solution to this puzzle is interesting-both pictures are correct, but not at the same time. The point is that one cannot be both a distant observer and a falling observer at the same time. If you are a distant observer, the information will be outside, and the interior spacetime must be viewed as non-existent since you can never access it even in principle (because of the existence of the horizon). On the other hand, if you are a falling observer, then you have the interior spacetime in which the information (the book itself) will fall, but this happens only at the cost of losing a part of spacetime in which Hawking radiation lies, which you can never access since you yourself are falling into the black hole. There is no inconsistency in either of these two pictures; only if you artificially "patch" the two pictures, which you cannot physically do, does the apparent inconsistency of information duplication occur. This somewhat surprising aspect of a system with gravity is called black hole complementarity, pioneered by 't Hooft, Susskind, and their collaborators [6].

\section{The Quantum Multiverse}

What does this discussion of black holes have to do with cosmology, in particular the eternally inflating multiverse? In 2011, it was suggested that quantum mechanics, especially its many world nature, plays an important role in addressing the problem of predictivity in the multiverse [7]. Contrary to the naive expectation, quantum mechanics is playing a crucial role in determining the global structure of the multiverse at the largest distance scales. In particular, it was argued that the eternally inflating multiverse and quantum mechanical many worlds a la Everett are the same thing:

\section{Multiverse $=$ Quantum Many Worlds}

in a certain specific sense. In this picture, the global spacetime of general relativity appears only as a derived concept at the cost of overcounting the true degrees of freedom of the theory; in particular, the infinitely large space associated with eternal inflation is a sort of "illusion." A "true" description of the multiverse must be intrinsically probabilistic in a quantum mechanical sense-probabilities in cosmology and quantum measurements have the same origin.

Let us describe this picture in somewhat more details. In cosmology, our space is generally surrounded by a cosmological horizon. The situation, therefore, is the "inside out" version of the black hole case viewed from a distant observer. As in the case of the black hole, quantum mechanics requires that spacetime on the other side of the horizon-in this case the exterior to the cosmological horizon - must be viewed as non-existent. In more technical terms, a quantum state describing the system represents only the region within the horizon, so that there is no infinite space in any single, consistent description of the system.

If a quantum state represents only space within the horizon, then where is the multiverse, which we thought exists in an eternally inflating space further away from our own horizon? The answer is-probability! The process of creating bubble universes is probabilistic in the quantum mechanical sense as it occurs through quantum tunneling. This implies that, starting from some initially inflating space, we could end up with different universes probabilistically. All the different 
universes, including our own, live in probability space. In technical terms, a state representing eternally inflating space evolves into a superposition of terms — or branches - representing different universes, but with each of them representing only the region within its own horizon. Note that there is no concept of infinitely large space here, which led to the ill-definedness of probability. The picture of an infinitely large multiverse, naively suggested by general relativity, appears only after "patching" pictures based on different branches together; but this vastly overcounts true degrees of freedom as was the case if we included both the interior spacetime and Hawking radiation in our description of a black hole.

The description of the multiverse presented here provides complete unification of the eternally inflating multiverse and Everett's many worlds in quantum mechanics. Suppose the multiverse starts from some initial state $\left|\Psi\left(t_{0}\right)\right\rangle$. This state evolves into a superposition of terms where various bubble universes nucleate in various locations. As time passes, a term representing each universe further evolves into a superposition of states representing various possible cosmic histories, including different outcomes of "experiments" performed within that universe. (These "experiments" may, but need not, be scientific experiments; they can be any physical processes.) At late times, the multiverse state $|\Psi(t)\rangle$ will thus contain an enormous number of terms, each of which represents a possible world that may arise from $\left|\Psi\left(t_{0}\right)\right\rangle$ consistently with the laws of physics. Probabilities in cosmology and microscopic processes are then both given by quantum mechanical probabilities in the same manner. The multiverse and quantum many worlds are the same thing - they simply refer to the same phenomenon occurring at (vastly) different scales.

\section{Future Prospects}

Where could future study go? An obvious direction is to look for further observational evidence of the multiverse. For this, we may look for phenomena that are hard to understand in the conventional thinking but can naturally occur in the multiverse. For example, the multiverse can naturally lead to a small nonzero negative spatial curvature in our universe. The current observational upper bound on the curvature is about $\left|\Omega_{\mathrm{K}}\right| \lesssim 0.005$, which can be improved to the level of 0.0001 in the next few decades. If a nonzero value is found, it would be difficult to understand it in the framework of conventional, non-multiverse cosmology. Interestingly, a discovery of a wrong sign, i.e. positive, curvature would falsify the multiverse as we understand it now, and exploration of curvature might also give us information about possible measures in the multiverse [8].

On a more theoretical side, we might ask further, even more fundamental questions. What is the quantum state of the multiverse? How is it selected? What is time? How does it emerge? The picture presented here does not solve all these problems in eternally inflating cosmology. It, however, does provide a framework to address these deep questions. As an example, I have considered the possibility that the static state of the multiverse, in the sense of Wheeler-DeWitt theory, may be selected uniquely by the normalizability condition in a quantum gravitational Hilbert space [9]. This, if successful, would provide the origin of time as we observe it in our universe.

It is fascinating that physicists can talk about big and deep questions like the ones discussed here based on concrete theoretical progress. No one really knows where these explorations will finally lead us. However, it seems quite likely that we live in an exciting era in which our scientific explorations reach beyond what we thought to be the entire physical world, our universe. 


\section{References}

[1] See, for example, A. Linde, "A brief history of the multiverse," Rept. Prog. Phys. 80, 022001 (2017) [arXiv:1512.01203 [hep-th]].

[2] For reviews, e.g., C. J. Hogan, "Why the universe is just so," Rev. Mod. Phys. 72, 1149 (2000) [astro-ph/9909295]; L. J. Hall and Y. Nomura, "Evidence for the multiverse in the standard model and beyond," Phys. Rev. D 78, 035001 (2008) [arXiv:0712.2454 [hep-ph]].

[3] S. Weinberg, "Anthropic bound on the cosmological constant," Phys. Rev. Lett. 59, 2607 (1987); see also, T. Banks, "T C P, quantum gravity, the cosmological constant and all that...," Nucl. Phys. B 249, 332 (1985); A. D. Linde, “The inflationary universe,” Rept. Prog. Phys. 47, 925 (1984).

[4] R. Bousso and J. Polchinski, "Quantization of four form fluxes and dynamical neutralization of the cosmological constant," JHEP 06, 006 (2000) [hep-th/0004134]; S. Kachru, R. Kallosh, A. Linde and S. P. Trivedi, "De Sitter vacua in string theory," Phys. Rev. D 68, 046005 (2003) [arXiv:hep-th/0301240]; L. Susskind, "The Anthropic landscape of string theory," in Universe or Multiverse?, ed. B. Carr (Cambridge University Press, 2007), p. 247 [hep-th/0302219].

[5] A. H. Guth, "Inflation and eternal inflation," Phys. Rept. 333, 555 (2000) [astro-ph/0002156].

[6] G. 't Hooft, "On the quantum structure of a black hole," Nucl. Phys. B 256, 727 (1985); “The black hole interpretation of string theory," Nucl. Phys. B 335, 138 (1990); L. Susskind, L. Thorlacius and J. Uglum, "The stretched horizon and black hole complementarity," Phys. Rev. D 48, 3743 (1993) [hep-th/9306069].

[7] Y. Nomura, "Physical theories, eternal inflation, and the quantum universe," JHEP 11, 063 (2011) [arXiv:1104.2324 [hep-th]]; "Quantum mechanics, spacetime locality, and gravity," Found. Phys. 43, 978 (2013) [arXiv:1110.4630 [hep-th]]; R. Bousso and L. Susskind, "The multiverse interpretation of quantum mechanics,” Phys. Rev. D 85, 045007 (2012) [arXiv:1105.3796 [hep-th]].

[8] A. H. Guth and Y. Nomura, "What can the observation of nonzero curvature tell us?," Phys. Rev. D 86, 023534 (2012) [arXiv:1203.6876 [hep-th]]; see also, M. Kleban and M. Schillo, "Spatial curvature falsifies eternal inflation," JCAP 06, 029 (2012) [arXiv:1202.5037 [astro-ph.CO]].

[9] Y. Nomura, “The static quantum multiverse," Phys. Rev. D 86, 083505 (2012) [arXiv:1205.5550 [hep-th]]. 